Reprod. Nutr. Dévelop., 1987, 27 (4), 801-810.

\title{
Effet de l'addition d'un " concentré " riche en fibres dans une ration à base de foin, distribuée à deux niveaux alimentaires chez la lapine adulte. 2. Mesures de digestibilité
}

\section{T. GIDENNE}

Laboratoire de Recherches sur I'Elevage du Lapin, I.N.R.A., B.P. 27, 31326 Castanet Tolosan Cedex, France.

Summary. Addition of a fibre-rich concentrate to a hay-based diet offered at two levels of feeding to adults rabbits. 2. Digestibility measurements.

The effect of adding a fibre-rich concentrate (wheat bran or beet pulp) to a hay-based diet (cock's foot-lucerne : 50/50) on the apparent digestibility of diets was measured in three groups of 8 adult doe rabbits (New-Zealand White $\times$ Californian). Diet composition was as follows : $90 \%$ hay only (diet F), hay + bran (diet FS) or hay + pulp (diet FP). Two levels of feeding were tested : ad libitum and $2 / 3$ of ad libitum intake.

Addition of wheat bran and especially of beet pulp led to a significant increase in the apparent digestibility of the whole diet. Our results confirm the high digestibility of cell-wall constituents of beet pulp : the apparent digestibility coefficient of hemicellulose in diet FP reached $60 \%$ (versus $23 \%$ for diet $F$ ) and that of cellulose $45 \%(21 \%$ for diet $F)$. Wheat bran supplied digestible hemicellulose (ADC $=48 \%$ for diet FS) while the apparent digestibility of bran cellulose was not higher than that of hay $(A D C=22 \%)$. This digestive interaction between raw material was accompanied by an interaction with the digestive transit. The less digestible diet (diet F) exhibited the shortest mean residence time (MRT). However, diet FS exhibited the longest residence time whereas it was less digestible than diet FP. These results suggest that, in the rabbit, wheat bran is a transit-regulator as already observed in other species.

Measuring apparent digestibility between the 10th and the 16th day of the feed restriction was too early for our doe rabbits. Values measured were not significantly different from those obtained during ad libitum feeding, although the MRT of diets was longer during restricted feeding.

\section{Introduction.}

Les relations entre la composition en constituants pariétaux et la valeur nutritive d'un aliment pour le lapin font l'objet de nombreuses études. Ainsi, il semble que la teneur en énergie digestible d'un aliment soit fortement liée à sa teneur en lignocellulose Van Soest (Battaglini et Grandi, 1984 ; Fraga et al., 1984). Mais les matières premières composant une ration ont souvent des origines végétales très diverses. De ce fait, peu d'études ont analysé les éventuelles interactions digestives 
entre les différentes matières premières d'un aliment, en particulier au plan de l'utilisation digestive des fibres.

Dans ce but, nous avons ajouté un « concentré » riche en fibres, son ou pulpe de betteraves, à une ration de base constituée de $90 \%$ de foin. Ces matières premières différant par leur composition en constituants pariétaux sont souvent incorporées en tant que source de fibres dans les aliments pour lapin. Notre étude se propose de mesurer l'effet de la nature du "concentré " sur la digestibilité de la ration et des constituants pariétaux des deux principaux composants : foin et " concentré ».

D'autre part, la digestibilité de la ration est améliorée par la restriction alimentaire selon Lebas (1979) et Ledin (1984a et b). Mais ces études ont été réalisées avec des lapines gestantes ou des lapereaux recevant des régimes dont la teneur en fibres était peu élevée ( $15 \%$ de cellulose brute). Pour vérifier cet effet de la restriction alimentaire sur la digestion, nous avons réalisé une mesure de digestibilité de rations riches en fibres (plus de $20 \%$ de lignocellulose) à deux niveaux d'alimentation, chez la lapine adulte non gestante.

\section{Matériel et méthodes.}

Aliments et matériel animal. - Trois rations ont été expérimentées. Leur composition chimique et centésimale figure au tableau 1. Ces rations, très riches en constituants pariétaux $(48,0$ à $54,5 \%$ de NDF), diffèrent surtout par leur teneur en cellulose Van Soest : 17,2 à $28,0 \%$.

La ration "Foin » est composée à $90 \%$ d'un foin de luzerne et dactyle (50/50). Dans les deux autres rations, le foin a été mélangé dans un rapport de

TABLEAU 1

Composition des aliments expérimentaux.

\begin{tabular}{lccc}
\hline \multicolumn{1}{c}{ Composants (\% MS) } & $\begin{array}{c}\text { Foin } \\
(\mathrm{F})\end{array}$ & $\begin{array}{c}\text { Foin }+ \text { Son } \\
(\mathrm{F}+\mathrm{S})\end{array}$ & $\begin{array}{c}\text { Foin + Pulpe } \\
(\mathrm{F}+\mathrm{P})\end{array}$ \\
\hline Foin (luzerne-dacyle, 50/50) & 90 & 36,0 & 36,0 \\
Son de blé tendre & - & 54,0 & - \\
Pulpe de betteraves & - & 4,0 & 54,0 \\
Farine de poisson " 72 " & 4,0 & 4,5 & 4,0 \\
Amidon de maïs & 4,5 & 0,5 & 0,5 \\
NaCl & 0,5 & 1,0 & 1,0 \\
CMV & 1,0 & & \\
\hline Analyse chimique & & 91,2 & 91,6 \\
Matière sèche & 91,8 & 8,1 & 7,9 \\
Matière minérale (\% MS) & 9,2 & 460 & 4244 \\
Energie brute (Kcal/kg MS) & 402 & 17,1 & 14,0 \\
Protéines brutes (N $\times 6,25)$ (\% MS) & 17,0 & 51,9 & 48,0 \\
"NDF" (\% MS) & 54,5 & 22,8 & 28,6 \\
"ADF" & 35,8 & 29,1 & 19,4 \\
Hémicelluloses Van Soest (\% MS) & 18,7 & 17,2 & 23,6 \\
Cellulose Van Soest (\% MS) & 28,0 & 5,6 & 5,0 \\
Lignines Van Soest (\% MS) & 7,8 & & \\
\hline
\end{tabular}


$2 / 3$ à un aliment "concentré " riche en parois : la pulpe de betteraves (lot foinpulpe) ou le son de blé tendre (lot foin-son). Par conséquent, les constituants pariétaux des rations ne proviennent que d'une ou deux matières premières : foin ou concentré. Ces dernières ont été broyées, mélangées aux autres éléments de la ration puis agglomérées sous forme de granulés de $3,5 \mathrm{~mm}$ de diamètre.

$\mathrm{Au}$ cours de la première période (P1), les rations ont été distribuées à volonté. Pendant une seconde période (P2), le niveau d'ingestion a été restreint individuellement aux $2 / 3$ de la consommation à volonté, mesurée durant la semaine précédant $\mathrm{P} 2$.

Les aliments ont été distribués à trois lots de 8 lapines adultes, métis (Néozélandais blanc $\times$ Californiens), maintenues en cages à digestibilité.

Controles zootechniques et mesures de digestibilité. - La consommation d'aliment a été contrôlée tous les deux jours pendant $P 1$, et quotidiennement pendant P2. Les poids vifs ont été contrôlés en début d'essai et à la fin de chaque période de mesures de digestibilité. Ces dernières ont été effectuées après une période d'accoutumance au régime de $15 \mathrm{j}$ pendant la période 1 et de $10 \mathrm{j}$ pendant la période 2. Pendant $6 \mathrm{j}$ consécutifs, les quantités d'aliments consommées et les quantités de fèces excrétées ont été mesurées quotidiennement. Après pesée des fèces fraîches, celles-ci ont été conservées en sachet étanche à $-18^{\circ} \mathrm{C}$, en vue de leur analyse.

Dosage. - La teneur en matière sèche des aliments et des fèces a été déterminée après dessiccation durant $24 \mathrm{~h}$ à $103^{\circ} \mathrm{C}$; leur teneur en matière minérale a été déterminée après calcination durant $5 \mathrm{~h}$ à $550^{\circ} \mathrm{C}$.

Sur les produits secs, la teneur en énergie (calorimètre adiabatique Parr) et la teneur en matières azotées totales $(\mathrm{N}$ Kjeldhal $\times 6,25)$ ont été également mesurées. Le dosage des constituants pariétaux a été réalisé semi-automatiquement selon la méthode de Van Soest et Wine (1967), modifiée par Giger et al. (1979).

Exploitation des résultats. - L'analyse statistique des données a été effectuée par analyse de variance à deux facteurs de classification : effet du type d'aliment + effet du niveau alimentaire + interaction entre les deux facteurs. Les moyennes sont comparées deux à deux par la méthode du $t$ corrigé dite de Bonferroni (Bachacou et al., 1981).

\section{Résultats.}

\section{Poids vifs et consommation.}

Le poids vif moyen des animaux (environ $4 \mathrm{~kg}$ ) n'a pas significativement différé entre les trois lots (tabl. 2), que ce soit en période d'alimentation à volonté (P1) ou en période d'alimentation restreinte (P2). Par contre, l'ingestion volontaire d'aliment (P1) a varié significativement selon la ration : l'aliment à base de foin et de son $(F+S)$ étant sous-consommé (106 g MS/j) par rapport à l'aliment à base de foin et de pulpe (137 $\mathrm{g} \mathrm{MS}_{/} / \mathrm{j}$ ) ou de foin seul (145 g MS/j). 
Durant la phase d'alimentation restreinte, le niveau alimentaire a été fixé à $66 \%$ de l'ingestion volontaire (g MS/ $\mathrm{kg}$ poids vif) constatée la semaine précédente. Mais par rapport à l'ingestion constatée pendant la semaine de mesures de digestibilité, ce niveau a été respectivement de 73,$1 ; 76,6$ et $69,0 \%$, pour les lots "foin " (F), "foin + son " (F + S) et « foin + pulpe " (F + P).

TABLEAU 2

Poids vifs et ingérés moyens $(x \pm \sigma)$ des lapines en fonction de la ration et du niveau alimentaire.

\begin{tabular}{|c|c|c|c|c|c|c|}
\hline & \multicolumn{3}{|c|}{ Ad libitum } & \multicolumn{3}{|c|}{ Rationné } \\
\hline & $n$ & $\begin{array}{l}\text { Poids vif } \\
\text { (g) }\end{array}$ & $\begin{array}{c}\text { Ingéré } \\
\text { (g MS/kg P. vif/j) }\end{array}$ & $n$ & $\begin{array}{l}\text { Poids vif } \\
\text { (g) }\end{array}$ & $\begin{array}{c}\text { Ingéré } \\
\text { (g MS/kg P. vif/j) }\end{array}$ \\
\hline Lot Foin & 8 & $\begin{array}{r}3857 \\
\pm 542\end{array}$ & $\begin{array}{r}37,5^{\mathrm{a}} \\
+4,8\end{array}$ & 8 & $\begin{array}{r}3749 \\
\pm 509\end{array}$ & $\begin{array}{r}27,4^{a} \\
\pm 0,7\end{array}$ \\
\hline $\begin{array}{l}\text { Lot Foin }+ \text { Son } \\
(F+S)\end{array}$ & 8 & $\begin{array}{r}4048 \\
\pm 369\end{array}$ & $\begin{aligned} & 26,6^{\mathrm{b}} \\
\pm & 10,3\end{aligned}$ & 8 & $\begin{array}{r}3920 \\
\pm 415\end{array}$ & $\begin{array}{c}20,4^{\mathrm{b}} \\
\pm 1,2\end{array}$ \\
\hline $\begin{array}{l}\text { Lot Foin }+ \text { Pulpe } \\
(F+P)\end{array}$ & 6 & $\begin{array}{r}4242 \\
\pm 404\end{array}$ & $\begin{array}{l}32,2^{\mathrm{ab}} \\
\pm 1,9\end{array}$ & 8 & $\begin{array}{r}3962 \\
\pm 303\end{array}$ & $\begin{array}{r}22,2^{\mathbf{c}} \\
\pm 1,2\end{array}$ \\
\hline F lot & & NS & * & & NS & ** \\
\hline
\end{tabular}

$(a, b, c)$ : Dans une même colonne, les moyennes affectées d'une lettre différente diffèrent au seuil $P<0,05$. significatif.

** : écart significatif au seuil $P<0,01$; * : écart significatif au seuil $P<0,05$; NS : écart non

Digestibilité des aliments.

Période d'alimentation à volonté (P1). - L'effet moyen du type d'aliment a été très significatif sur les valeurs de tous les CUDa, excepté celles des lignines (tabl. 3). Nous n'avons pas observé d'interaction significative entre l'effet du type d'aliment et l'effet du rationnement, quel que soit le CUDa mesuré. La digestibilité apparente de la matière sèche (MS) et de la matière organique (MO), peu élevée dans le cas du lot $F(47,4$ et $46,1 \%)$ est significativement améliorée de 13 et $26 \%$ pour les lots $F+S$ et $F+P$. La digestibilité de l'énergie est améliorée de 23 et $36 \%$ respectivement quand le son ou la pulpe se substituent au foin. La teneur en énergie digestible (ED) des aliments $F, F+S, F+P$ est donc estimée à 1998,2497 et $2623 \mathrm{kcal} / \mathrm{kg}$ MS. Par ailleurs, la digestibilité des protéines brutes (MAT) a diminué de $19 \%$ quand la pulpe a été substituée à une partie du foin, mais elle n'a pas différé entre les lots $F$ et $F+S$.

Lorsque le son a remplacé une fraction du foin, la digestibilité des « parois totales " (NDF) est passée de 23,5 à 35,2 \% (+ $50 \%$ ). Cette hausse de la digestibilité apparente des parois est essentiellement due à une meilleure digestion des hémicelluloses $(+54 \%)$. Ce phénomène est accentué lorsque la pulpe de betterave remplace le foin : le CUDa de "NDF " est double. Dans ce cas, la hausse de la digestibilité apparente des parois totales provient d'une meilleure digestibilité des hémicelluloses $(+96 \%)$, mais aussi de la cellulose $(+114 \%)$. 


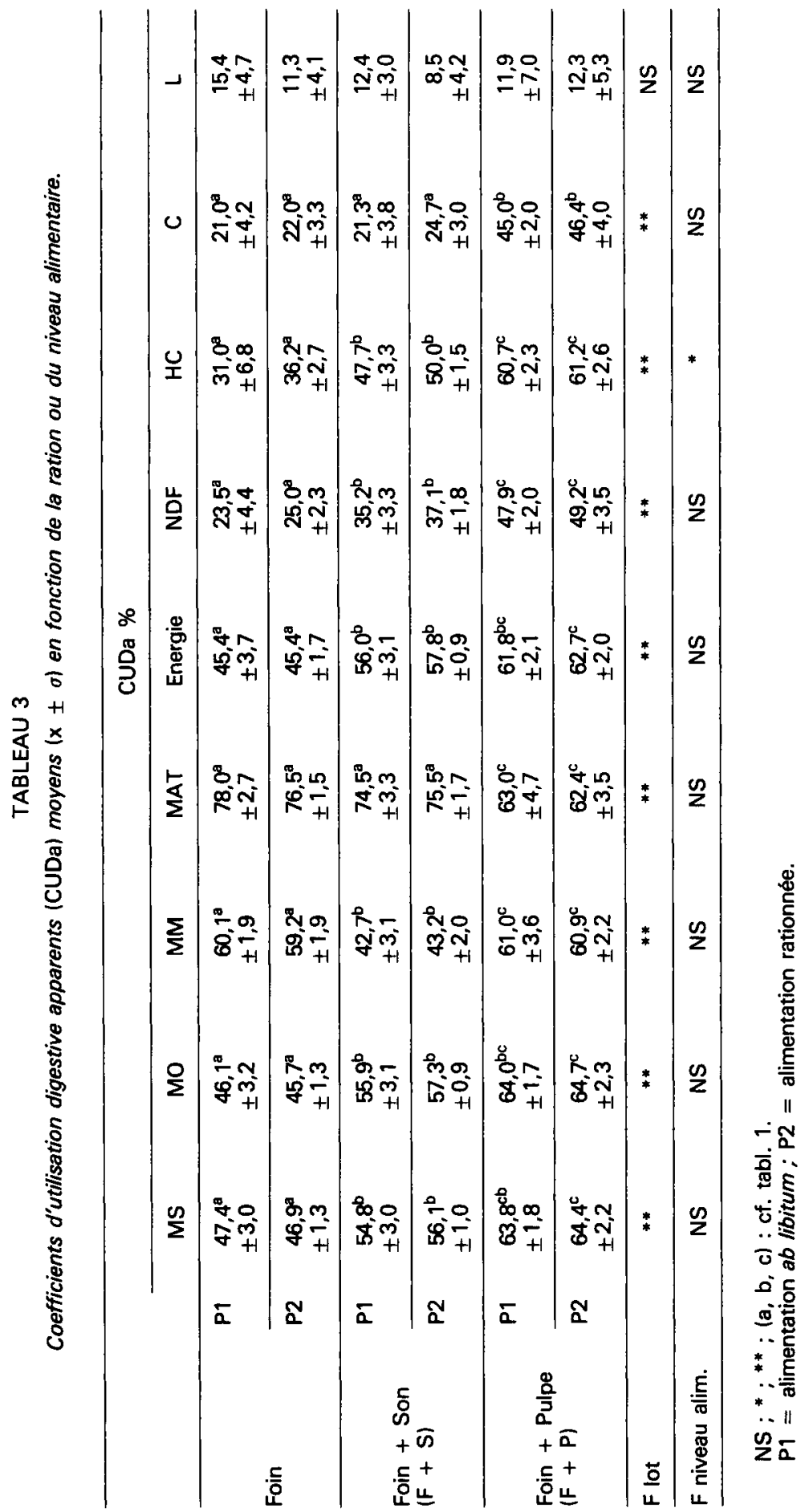

Reproduction, Nutrition, Développement $n^{\circ} 4$, vol. 27 (1987). - 5 
C'est dans le cas des CUDa des constituants pariétaux que les variations interindividuelles ont été les plus élevées. La digestibilité apparente des lignines qui est en moyenne de $12 \%$ présente les coefficients de variation les plus importants (24 à $59 \%$ ).

Période d'alimentation rationnée (P2). - Seule la digestibilité apparente des hémicelluloses a été significativement améliorée par la baisse du niveau alimentaire (tabl. 3). Toutefois, nous avons pu observer une légère hausse (non significative) de la digestibilité apparente des constituants pariétaux (excepté les lignines) lors de la période 2, pour les trois rations. De plus, dans le cas de la ration foin-son, c'est la digestibilité de l'ensemble des constituants qui semble être améliorée par la restriction alimentaire.

Digestibilité apparente recalculée des constituants pariétaux des matières premières.

Si on considère constante la digestibilité des constituants pariétaux de la ration à base de foin seul, il est alors possible de calculer, par substitution, celle des aliments son et pulpe (tabl. 4).

Ainsi, le son semble constituer un apport d'hémicelluloses très digestibles pour le lapin ; tandis que la cellulose ne paraît pas plus digestible que celle provenant du foin.

Dans le cas de la pulpe de betteraves, la digestibilité des parois serait extrêmement élevée. Elle dépasserait $100 \%$ dans le cas des hémicelluloses, pendant la période 1. Notre hypothèse de départ, postulant une digestibilité constante pour le foin, n'est donc pas vérifiée. II y aurait donc une interaction positive au plan de la digestion des parois lors du mélange de matières premières tel que foin et pulpe de betteraves.

TABLEAU 4

Digestibilité apparente recalculée (CUDa \%) moyenne des constituants pariétaux du son et de la pulpe de betteraves en fonction du niveau alimentaire.

\begin{tabular}{lccccc}
\hline & & NDF & Hémicelluloses & Cellulose & Lignines \\
\hline \multirow{2}{*}{ Son de blé } & $\mathrm{P} 1$ & 52,7 & 72,7 & 21,7 & 7,9 \\
\cline { 2 - 6 } & $\mathrm{P} 2$ & 55,2 & 70,7 & 28,7 & 4,3 \\
\hline \multirow{2}{*}{ Pulpes de betteraves } & $\mathrm{P} 1$ & 84,5 & 105,2 & 81,0 & 6,6 \\
\cline { 2 - 6 } & $\mathrm{P} 2$ & 85,5 & 98,7 & 83,0 & 13,8 \\
\hline
\end{tabular}

P1, P2 : cf. tabl. 3.

\section{Discussion.}

\section{Effet du type d'aliment.}

Compte tenu de la haute teneur en fibres de nos rations $150 \%$ NDF en moyenne), les quantités ingérées sont relativement élevées, excepté le cas de l'aliment contenant le son ( $F+S$ ). Chez le lapin en croissance, la quantité d'aliment 
ingéré est liée négativement à la teneur en énergie digestible (ED) (Lebas, 1975 ; Lebas et al., 1982). Ceci est vérifié si l'on considère les régimes $F$ et $F+P$. II semblerait que le régime $F+S$ ait manqué d'appétence, $d$ 'où une sous-consommation qui ait subsisté même après deux semaines d'accoutumance à l'aliment. En effet, Beltran et al. (1984) n'observent pas d'écart chez des lapereaux recevant des rations contenant $1 / 3$ de son ou de pulpe.

$D$ 'autre part, la teneur en ED du régime est liée négativement à la teneur en lignocellulose (ADF) ; ceci est observé chez les lapins adultes (Martinez et Fernandez, 1980 ; Hoover et Heitmann, 1972 ; Battaglini et Grandi, 1984), ou en croissance (Fraga et al., 1984). Pour nos régimes, cette relation n'est pas vérifiée ; par contre, nous observons une relation négative entre ED et teneur en NDF de nos aliments.

Quels que soient la ration ou le niveau alimentaire, la digestibilité des hémicelluloses est supérieure à celle de la cellulose. Ceci avait déjà été observé chez le lapin adulte avec un aliment à base de foin de fléole (Uden et Van Soest, 1982), mais aussi chez le rat et le porc alimentés avec des régimes à base de foin ou de son de blé (Keys et al., 1970 ; Bertrand et al., 1981).

En accord avec la bibliographie (Candau et al., 1978; Lebas et Cheriet, 1981 ; Nogradi, 1984), nos résultats montrent la bonne valeur énergétique de la pulpe de betterave pour le lapin et la digestibilité élevée de ses constituants pariétaux. Cette dernière provient non seulement de la fraction hémicellulose, mais aussi de la cellulose. Par ailleurs, nous signalons une digestibilité peu élevée des protéines contenues dans les pulpes, en accord avec les résultats de Maertens et De Groote (1984). D'autres travaux indiquent par contre une digestibilité des protéines plus élevée, comparable à celle de la luzerne (Martinez et Fernandez, 1980 ; Evans et al., 1983 ; Beltran et al., 1984). Les pulpes de betteraves montrent donc des variations assez importantes de qualité (composition azotée, en sucres solubles, ....), sans doute en fonction de leur origine et plus particulièrement de leur technique de production.

Selon Beltran et al. (1984), le remplacement de pulpes de betteraves (32\% de la ration) par du son de blé entraîne une hausse de la digestibilité de la lignocellulose (ADF). Cette dernière, mesurée chez des lapins en croissance, demeurait toutefois très peu élevée : $10 \%$. Nos résultats indiquent chez l'adulte, une digestibilité de l'ADF d'un niveau supérieur. Lorsque le son remplace le foin, le CUDa de l'ADF ne varie pas $(19,4 \%)$. Mais lorsque la pulpe se substitue au foin, la digestibilité apparente de l'ADF atteint 39,2\%. Ledin (1984b) observe également une hausse de digestibilité pour la lignocellulose, quand la pulpe de betteraves remplace du son de blé.

La digestibilité apparente (recalculée) des hémicelluloses de la pulpe et du son est très supérieure à celles classiquement observées dans des travaux antérieurs (Candau et al., 1978 ; Randall, 1977 ; ...). Elle dépasse $100 \%$ dans le cas des pulpes de betteraves (période 1). Cela montre que l'on ne peut considérer constante la digestibilité du foin, indiquant ainsi une interaction digestive positive entre foin et concentré riche en parois. Cette interaction au plan de l'utilisation digestive, se manifeste également sur les valeurs des temps de séjour moyen 
(TSM) du foin et du " concentré " qui ont été mesurées sur ces mêmes lots d'animaux (Gidenne et al., 1987).

Ainsi, l'aliment « foin seul » est le plus mal digéré et il a le TSM le plus bref : $14,8 \mathrm{~h}$. Pour les deux autres aliments, les particules "marquées » de foin et de " concentré " ont un TSM similaire, bien que la digestibilité de leurs constituants pariétaux soit différente. L'addition de concentré élève ainsi le TSM des particules de foin de $4 h$ et $2 h$, respectivement pour les lots " $F+S$ " et « $F+P$ ". Lebas et Laplace (1977) constatent que l'aliment le plus digestible est le moins consommé et qu'il présente le transit le plus lent. Mais pour nos aliments riches en fibres, la ration foin + son fait l'objet du transit le plus lent alors qu'elle est moins digestible que la ration foin-pulpe. Le temps de séjour élevé de la ration " $F+S$ " pourrait être lié à son faible niveau d'ingestion. Mais en période 2 (rationnement), le niveau d'ingestion entre les rations " $F+S$ " et " $F+P$ " est similaire, et les écarts de TSM sont toujours aussi élevés (Gidenne et al., 1987). II convient de distinguer pour nos matières premières leur valeur digestive et leur effet sur la vitesse de transit de la ration. L'effet régulateur du son de blé sur le transit digestif a été montré chez d'autres espèces monogastriques (rat, porc, homme) ; ainsi, le son de blé rétablirait un transit normal chez des individus ayant un transit trop long ou trop court (Ruckebusch et al., 1981). Dans le cas de nos rations riches en fibres occasionnant un transit rapide, le son de blé jouerait donc aussi ce rôle régulateur.

\section{Effet du rationnement.}

La restriction alimentaire entraîne une meilleure digestibilité de la ration, chez le lapin en croissance (Arkhurst, 1973; Ledin, 1984a, 1984b) ou chez la lapine adulte gestante (Lebas, 1979). Nos résultats indiquent que seules les hémicelluloses sont significativement mieux digérées lors du rationnement. Les mesures de transit réalisées sur ces animaux ( $3 \mathrm{j}$ après la période de mesure de digestibilité) indiquent pourtant un allongement du temps moyen de séjour : $+15,+26$, $+21 \%$ respectivement pour les lots $F, F+S$ et $F+P$ (Gidenne et al., 1987). Par ailleurs, les animaux du lot $F+S$ présentent les écarts de digestibilité entre $\mathrm{P} 1$ et $\mathrm{P} 2$ les plus élevés; c'est également sur ce lot que l'écart du TSM entre P1 et $\mathrm{P} 2$ est le plus important. Plusieurs paramètres peuvent rendre compte de ces faibles écarts de digestibilité entre les deux périodes. Ainsi, Arkhurst (1973) constate chez le lapin en croissance, une hausse du CUDa mesuré entre le 21e et le $35^{\mathrm{e}} \mathrm{j}$ de rationnement seulement. Dans notre cas, la mesure de digestibilité a été effectuée entre le $10^{\mathrm{e}}$ et le $16^{\mathrm{e}} \mathrm{j}$ de rationnement. Ce délai est peut-être trop court pour permettre une adaptation digestive des animaux. D'autre part, Ledin (1984b) signale que l'effet de la restriction alimentaire (60\% de l'ad libitum) sur la digestibilité est plus faible pour des lapins de $2,5 \mathrm{~kg}$ que pour des lapins de $1,5 \mathrm{~kg}$. Par conséquent, dans le cas de nos lapines adultes $(3,8$ à $4,2 \mathrm{~kg})$, le temps de rationnement ne semble pas suffisamment long pour observer une hausse significative de la digestibilité de la ration. 


\section{Conclusion.}

L'addition de son de blé et de pulpes de betteraves dans une ration à base de foin améliore significativement la digestibilité de la ration dans son ensemble. Cette meilleure efficacité digestive provient non seulement de la bonne valeur digestive du son et surtout de la pulpe, mais aussi d'une meilleure digestibilité du foin. Cette interaction entre matières premières au plan de leur utilisation digestive est associée à une interaction au plan de leur temps de séjour. La ration foin, la moins digestible, présente le temps de séjour moyen le plus bref. Ceci est donc en accord avec le principe classiquement admis, d'une relation positive entre TSM et digestibilité de la ration. A partir de nos essais, ce principe ne semble plus vérifié lorsqu'il s'agit de mélange de matières premières. Ainsi, la ration la plus digestible $(F+P)$ n'a pas le TSM le plus long. Le TSM élevé mesuré pour la ration " $F+S$ » semble indiquer chez le lapin le rôle régulateur du transit joué par le son de blé et qui a déjà été observé chez d'autres espèces monogastriques.

Une restriction alimentaire durant $10 \mathrm{j}$ n'a pas significativement amélioré l'efficacité digestive des lapines, alors que le transit digestif est notablement ralenti.

Ainsi la digestibilité d'un mélange de matière première ou d'une ration ne serait pas systématiquement fonction de leur temps de séjour dans le tube digestif. Pour prévoir la valeur alimentaire d'une matière première, en particulier si elle est riche en fibres, il serait donc utile de tenir compte des phénomènes d'interactions entre matières premières, au plan du transit et de la digestion.

Cette étude limitée aux interactions entre constituants pariétaux devra, à l'avenir, être complétée par des travaux concernant les interactions entre constituants pariétaux et constituants plus digestibles (azote, ...). Ceci permettrait de mieux prévoir la valeur alimentaire des rations complexes (mélanges de matières premières : sources de fibres ou " concentré ») destinées au lapin.

Reçu en novembre 1986. Accepté en mars 1987.

\section{Références}

ARKHURST G., 1973. Effet chez le lapin en croissance de l'addition de dl méthionine au régime alimentaire. Th. $3^{e}$ cycle, Univ., Paris VI.

BACHACOU J., MASSON J. P., MILLIER C., 1981. In Manuel de la programmatheque statistique, AMANCE 81. Ed. I.N.R.A.

BATTAGLINI M., GRANDI A., 1984. Stima del valore nutritivo dei mangimi per conigli. $3^{e}$ Congr. mond. Cunicult., Rome, Vol. 1, 252-264.

BELTRAN M. T., MARTINEZ J., FERNANDEZ J., CERVERA C., 1984. Estimacion del valor nutritivo de diversas matieras primas para conejos. 3e Congr. mond. Cunicult., Rome, Vol. 1, 265-272.

BERTRAND D., BRILLOUET J. M., RASPER V. F., BOUCHET B., MERCIER C., 1981 . Effects of rat digestion upon native, enzimically or chemically modified wheat brans and native oat bran. Cereal Chem., 58, 375-380. 
CANDAU M., BERTRAND B., FIORAMONTI J., 1978. Variations de la digestibilité des constituants de la ration chez le lapin. C. $R$. Soc. Biol., 172, 554-559.

EVANS E., JEBELLIAN V., RYCQUART W. C., 1983. Effect of partial replacement of fiber from alfalfa with fiber from other ingredients upon performances of fryer rabbits. $J$. appl. Rabb. Res., 6, 6-8.

FRAGA M. J., BARRENO C., CARABANO R., MENDEZ J., BLAS J. C. de, 1984 . Effecto de los niveles de fibra y proteina del pienso sobre la velocidad de crecimiento y los parametros digestivos de los conejos. An. Instit. nac. Investigat. agra., $n^{\circ} 21,91-110$.

GIDENNE T., PONCET C., GOMEZ L., 1987. Effet de l'addition d'un concentré riche en fibres dans une ration à base de foin, distribuée à deux niveaux alimentaires chez la lapine adulte. 1/temps de séjour des aliments. Repr. Nutr. Dévelop., 27, (sous presse).

GIGER S., SAUVANT D., DORLEANS M., MORAND-FEHR P., 1979. Détermination semi-automatique des constituants membranaires des aliments concentrés par la méthode de Van Soest. 30th Annu. Meet. Europ. Assoc. anim. Prod., Harrogate, England, 23-26 July.

HOOVER W. H., HEITMANN R. N., 1972. Effects of dietary fiber levels on weight gain, cecal volume and volatile fatty acid production in rabbits. J. Nutr., 102, 375-379.

KEYS J. E., VAN SOEST P. J., YOUNG E. F., 1970. Effect of increasing dietary cell-wall content on the digestibility of hemicellulose and cellulose in swine and rat. J. anim. Sci., 31, 1172-1177.

LEBAS F., 1975. Influence de la teneur en énergie de l'aliment sur les performances de croissance chez le lapin. Ann. Zootech., 24, 281-288.

LEBAS F., 1979. Efficacité de la digestion chez la lapine adulte. Effet du niveau d'alimentation et du stade de gestation. Ann. Biol. anim. Bioch. Biophys., 19, 969-973.

LEBAS F., CHERIET S., 1981. Utilisation de 3 sources de lest chez le lapin en croissance. Cuniculture, 40. 190-193.

LEBAS F., LAPLACE J. P., 1977. Le transit digestif chez le lapin. VIII/Influence de la source de cellulose. Ann. Zootech., 26, 575-584.

LEBAS F., LAPLACE J. P., DROUMENO P., 1982. Effet de la teneur en énergie de l'aliment chez le lapin. Variations en fonction de l'åge des animaux et de la séquence des régimes alimentaires. Ann. Zootech., 31, 233-256.

LEDIN I., 1984a. Effect of restricted feeding and realimentation on growth, carcass composition and organ growth during the first seven days of realimentation in rabbit. Acta. agric. scand., 34, $54-66$.

LEDIN I., 1984b. Effect of restricted feeding and realimentation on compensatory growth and organ growth in rabbit. Ann. Zootech., 33, 33-50.

MAERTENS L., De GROOTE G., 1984. Digestibility and digestible energy content of a number of feedstuffs for rabbits. $3^{3}$ Congr. mond. Cunicult., Rome, Vol. 1, 244-251.

MARTINEZ PASCUAL J., FERNANDEZ CARMONA J., 1980. Composition, digestibilidad, valor nutritivo y relaciones entre ambos de diversas piensos para conejos. $2^{\circ}$ Congr. mond. Cunicult., Barcelone, Vol. 2, 214-224.

NOGRADI S., GACS J., 1984. Examination of crude fiber's digestibility in rabbit nutrition. $3^{*}$ Congr. mond. Cunicult., Rome, Vol. 1, 475-484.

RANDALL K. E., 1977. Digestion of dietary fiber and energy in the adult New-Zealand white rabbit. Thesis, Aberdeen Univ., UK, $26 \mathrm{p}$.

RUCKEBUSCH Y., BUÉNO L., FIORAMONTI J., 1981. Constituants alimentaires et motricité digestive. Repr. Nutr. Dévelop., 21, 749-771.

UDEN P., VAN SOEST P. J., 1982. Comparative digestion of Timothy (Phleum-partense) fibre by ruminants, equines and rabbits. Br. J. Nutr., 47, 267-272.

VAN SOEST P. J., WINE R. H., 1967. Use of detergents in the analysis of fibrous feed. IV/Determination of plant cell-wall constituents. J. Ass. off. agric. Chem., 50, 50-55. 